\title{
Comparison of Label-Free ACh Image Sensors Based on CCD and LAPS
}

\author{
Shoko Takenaga ${ }^{1}$, Carl Frederik Werner ${ }^{2,3}$, Kazuaki Sawada ${ }^{1}$, Michael J. Schöning ${ }^{2,3}$ \\ ${ }^{1}$ Toyohashi University of Technology, 1-1 Hibarigaoka, Temaku-cho, Toyohashi, Aichi, Japan \\ f.werner@fh-aachen.de \\ ${ }^{2}$ Institute of Nano- and Biotechnologies (INB), FH Aachen, Campus Jülich, Heinrich-Mußmann-Str. 1 , \\ 52428 Jülich, Germany \\ ${ }^{3}$ Peter Grünberg Institute (PGI-8), Research Centre Jülich GmbH, 52525 Jülich, Germany
}

\begin{abstract}
:
Semiconductor-based chemical image sensors, like the $\mathrm{pH}$-image sensor based on a charge-coupled device (CCD) or the light-addressable potentiometric sensor (LAPS), are becoming a powerful tool for label-free imaging of biological phenomena. We have proposed a polyion-based enzymatic membrane to develop an acetylcholine (ACh)-image sensor for neural cell activity observations. In this study, a CCD-type ACh image sensor and a LAPS-type ACh image sensor were fabricated and the prospect of both sensors was clarified by making a comparison of their basic characteristics.
\end{abstract}

Key words: charge-coupled device, light-addressable potentiometric sensor, label-free imaging, acetylcholine, polyion complex membrane

\section{Introduction}

Chemical image sensors, like the $\mathrm{pH}$-image sensor based on a charge-coupled device (CCD) or the light-addressable potentiometric sensor (LAPS) are able to determine the $\mathrm{pH}$ value and its dynamic changes on the sensor surface in a spatial-resolved manner. Both sensors are semiconductor-based chemical sensors with an electrolyte/insulator/semiconductor (EIS)-layered structure. In case of the CCDtype set-up, each CCD spot is measuring the local $\mathrm{pH}$ value [1]. The CCDs and the signal processing circuits are fabricated by complementary metal-oxide-semiconductor (CMOS) technologies. For LAPS, a single EIS-structured sensor chip is used. By illuminating a local part of the semiconductor with a modulated light source, a photocurrent will be generated that depends on the local $\mathrm{pH}$ value of the sensor on that illuminated area $[2,3]$. In the present work, both sensor types should be compared in case of an acetylcholine (ACh)-image sensor. Therefore, the enzyme acetylcholine esterase (AChE) was immobilized on each sensor surface. Due to the ACh-AChE enzyme reaction, hydrogen ions will be generated that can be detected by the particular $\mathrm{pH}$-sensing surface. The final goal of this experiment is to investigate and image the ACh release in the synapse of neurons [4].

\section{Principle}

The principle of the proposed ACh-image sensors is based on the enzymatic reaction of AChAChE, specifically the oxidation of ACh according to the following reaction:

$$
\mathrm{ACh}+\mathrm{H}_{2} \mathrm{O} \stackrel{\text { AChE }}{\longrightarrow} \text { choline }+\mathrm{CH}_{3} \mathrm{COO}^{-}+\mathrm{H}^{+}
$$

The use of a polyion-complex enzyme membrane is one of the comprehensive methods to immobilize the enzyme on a substrate [5]. AChE is attached in the membrane by the electrostatic force of the polyanion (poly(L-lysine) hydrobromide) with a negative charge, and the polycation (poly(sodium-4-styrenesulfonate)) with a positive charge (see Fig. 1). The electrostatic force is achieved by the adjustment of number of charges into poly(L-lysine) hydrobromide and poly(sodium-4-styrenesulfonate) equivalently. This method has the advantage that the enzyme could be immobilized adjacent to the sensor at a high density

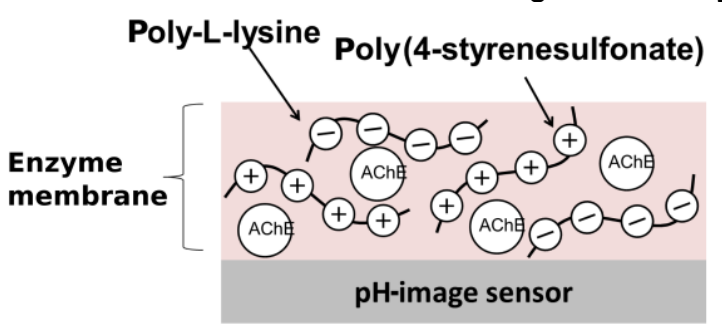

Fig. 1: Polyion-complex enzyme membrane including AChE on a $\mathrm{pH}$-image sensor. 
$(\sim \mu \mathrm{m})$. The proposed ACh-image sensor was fabricated by immobilizing the enzyme AChE on a $\mathrm{pH}$-image sensor as shown in Fig. 1. AChE converts ACh to choline and acetic acid, when ACh was hydrolyzed.

Due to the ACh-AChE enzymatic reaction, hydrogen ions will be released, which cause a $\mathrm{pH}$ change. This $\mathrm{pH}$ change results in a change of the surface potential $\Phi_{\mathrm{s}}$ at the electrolyte/insulator interface according to the sitebinding model $[6,7]$. This surface potential can be read out in a spatial-resolved manner by various sensor principles such as the CCD-type $\mathrm{pH}$-image sensor or the LAPS.

In case of the CCD-type set-up, the depth of the potential well in the semiconductor varied when it detects the change in the concentration of hydrogen ions generated by the $\mathrm{ACh}-\mathrm{AChE}$ enzyme reaction in the sensing area $\left(\mathrm{Si}_{3} \mathrm{~N}_{4}\right)$, as shown in Fig. 2. The output signal of the sensor depends on the ACh concentration in the solution. The $\mathrm{pH}$-image sensor chip was composed of a $32 \times 32$ pixel array and CMOS technique with horizontal and vertical shift registers. Each pixel was $130 \times 130 \mu^{2}$, with a sensing area of approximately $40{\mu \mathrm{m}^{2}}^{2}$.

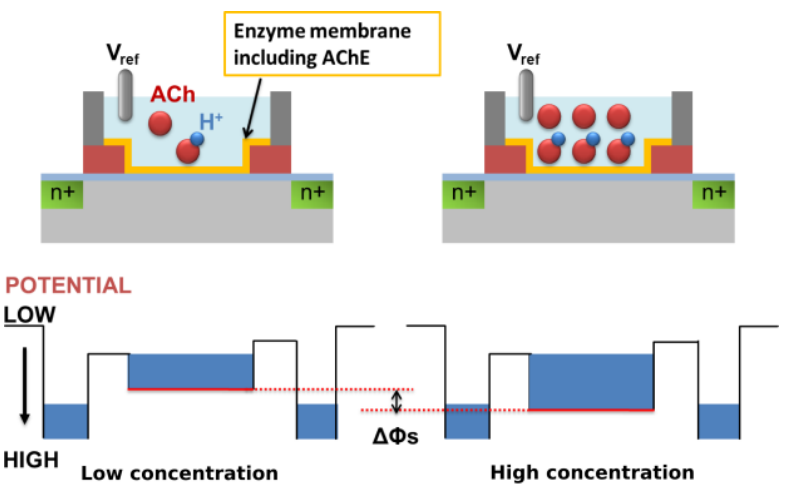

Fig. 2: Principle of the CCD-type ACh image sensor depicted for one measurement pixel.

In case of the LAPS-type set-up, a single EISstructured sensor chip with a sensing layer of $\mathrm{Ta}_{2} \mathrm{O}_{5}$ was used. The bias voltage as well as the surface potential will affect the local width of the space-charge region on the insulator/semiconductor interface. A light pointer is used to shine modulated light into the semiconductor and realize the addressability, as shown in Fig. 3. Depending on the width of the spacecharge region an external photocurrent will be generated. As light pointer a 4x4 infrared lightemitting diode (IR-LED) array is used for rearside illumination resulting in measurement spots with a radius of about $1.5 \mathrm{~mm}$. The surface potential is calculated by the measured photocurrent amplitude and a previously determined photocurrent/bias voltage curve and is given as sensor-output signal. More information about the applied LAPS set-up can be found elsewhere [2,3,7-11].

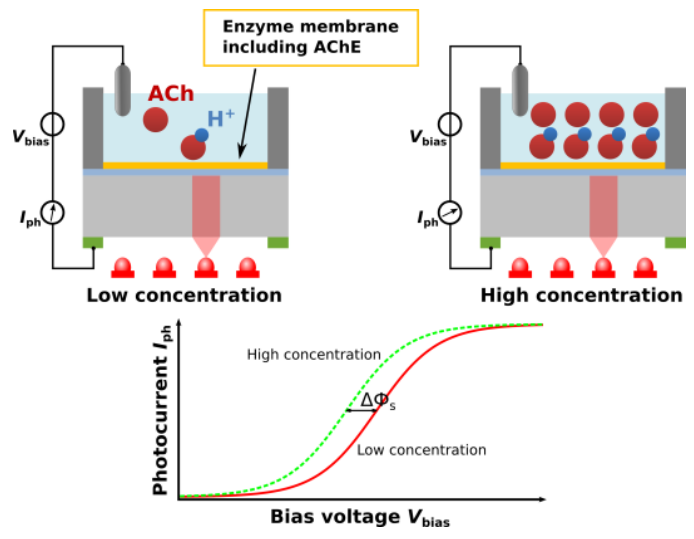

Fig. 3: Principle of the LAPS ACh image sensor.

\section{Experimental}

A CCD-type $\mathrm{pH}$ image sensor with $32 \times 32$ pixels and an active sensor size of $4 \times 4 \mathrm{~mm}^{2}$ [1] as well as an LAPS with a $4 \times 4$ IR-LED array and an active sensor size of $15 \times 15 \mathrm{~mm}^{2}$ [10] have been used. The enzyme AChE has been immobilized with the help of a polyion-based membrane. Three different aqueous solutions were prepared using $0.01 \mathrm{M}$ phosphate buffer solution (PBS), as follows: poly(L-lysine) hydrobromide (60 $\mathrm{mM}$ in monomer units), AChE $(100 \mathrm{kU} / 10 \mu \mathrm{l}), \quad$ and poly(sodium-4styrenesulfonate) ( $75 \mathrm{mM}$ in monomer units). The three solutions were mixed, and placed on the CCD-type and LAPS-type image sensor, respectively $(60 \mu \mathrm{l}$ and $1.08 \mathrm{ml})$. After that, the sensors were allowed to dry at room temperature for one day.

\section{Results}

Figure 4 shows a photograph of the two chosen sensor set-ups, that is the CCD-type enzymatic sensor (a) and the enzyme-based LAPS (b), respectively. In the experiments, the enzyme activity was varying from about 2 to 6 units $/ \mathrm{mm}^{2}$. The response of both sensor types towards different ACh concentrations from 1 to $100 \mathrm{mM}$ was investigated as calibration measurement (Fig. 5), which underlines the expected dependence. The different behavior in the signal amplitude of the sensor-output signal might be explained due to i) the different amplification of the output signal of both sensor types and ii) the varying enzyme activity on top of the particular sensor surface. The LAPS showed a more stable adhesion of the polyion-based enzymatic membrane, which is probably to the absolutely flat sensor surface of the LAPS chip compared to the more "rougher" surface (caused by the photolithographic patterning) of the CCD-type sensor. 


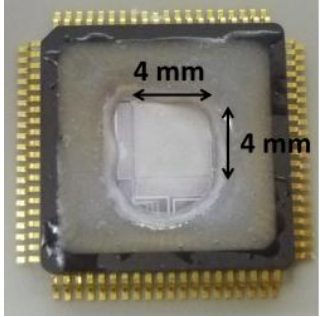

(a)

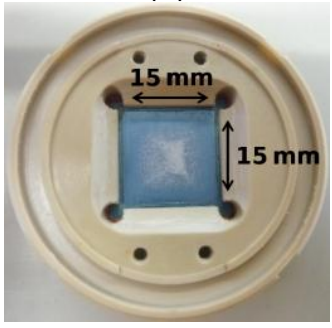

(b)

Fig. 4: Photographs of the ACh-image sensor based on (a) CCD and (b) LAPS.

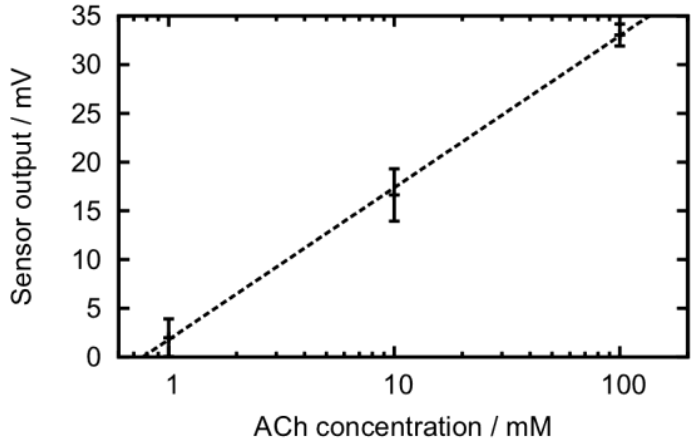

(a)

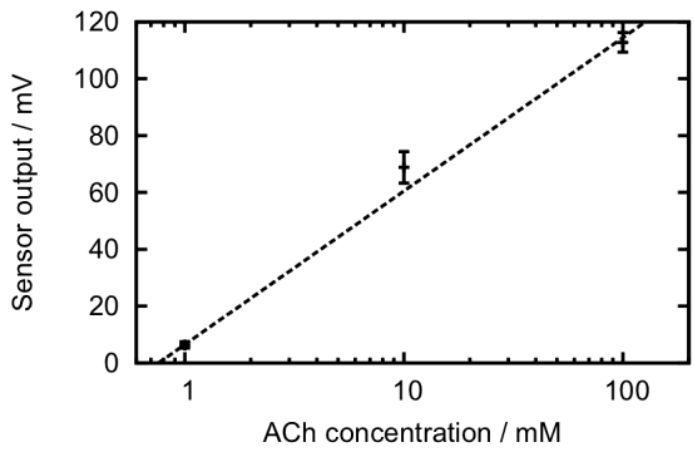

(b)
In addition, real-time (i.e., dynamic video) investigations with a measurement time of $200 \mathrm{~ms} /$ frame have been performed with both sensor types. Here, the diffusion of a $200 \mathrm{mM}$ ACh-containing droplet in PBS solution to the particular sensor surface and the subsequent catalytic conversion by the enzyme on top of the sensor surface has been monitored. The results demonstrated the ability of both sensor types to determine those changes spatially and time-resolved.

\section{Conclusions}

The CCD-type $\mathrm{pH}$-image sensor and the LAPS are able to detect ACh with the help of the enzyme AChE, immobilized in a polyion-based membrane. Both sensor types determine the analyte ACh spatially and time-resolved. A comparison of the basic characteristics of both sensor types is summarized in Tab. 1. The fabrication of the CCD-type sensor is rather complex, but all necessary signal-processing circuits are integrated on the sensor chip in CMOS technology. On the other hand, the LAPS chips are easy to fabricate, whereas the driving of the light sources and the signal processing of the photocurrent have to be done externally from the LAPS chip. Due to the fact, that the measurements spots of the LAPS are defined by external light sources, an alignment to an additional micro-fluidic set-up might be possible.

\section{Acknowledgements}

This work was partially supported by the Core Research for Evolutional Science and Technology (CREST) from Japan Science and Technology Agency (JST), an independent public body of the Ministry of Education, Culture, Sports, Science and Technology (MEXT).

The authors also thank the German Federal Ministry of Food, Agriculture and Consumer Protection (BMELV) and the "Fachagentur Nachwachsende Rohstoffe e.V." (FNR) for partial financial support of this work (Bio-LAPS).

Fig. 5: Sensor response of the ACh sensors to different ACh concentrations with (a) CCD and (b) LAPS set-up, respectively.

Tab.1: Properties of the ACh-image sensor for the CCD- and LAPS set-up.

\begin{tabular}{ccc}
\hline & CCD-type ACh image sensor & LAPS-type ACh image sensor \\
\hline Signal processing circuit & Integrated & External \\
$\begin{array}{c}\text { Complexity of readout and driving } \\
\text { Addressability }\end{array}$ & + & 0 \\
$\begin{array}{c}\text { Adherence of ACh polyion-based } \\
\text { membrane }\end{array}$ & Depending on fabrication & Depending on light sources \\
\hline
\end{tabular}




\section{References}

[1] T. Hizawa, K. Sawada, H. Takao, M. Ishida, Characteristics of highly sensitive $\mathrm{pH}$ sensors with charge accumulation operation, Japanese Journal of Applied Physics 45, 9259-9263 (2006); doi: 10.1143/JJAP.45.9259

[2] D. G. Hafeman, J. W. Parce, H. M. McConnell, Light-addressable potentiometric sensor for biochemical systems, Science 240, 1182-1185 (1988); doi: 10.1126/science.3375810

[3] J. C. Owicki, L. J. Bousse, D. G. Hafeman, G. L. Kirk, J. D. Olson, H. G. Wada, J. W. Parce, The light-addressable potentiometric sensor: Principles and biological applications, Annual Review of Biophysics and Biomolecular Structure 23, 87113 (1994); doi: 10.1146/annurev.bb.23.060194.000511

[4] S. Takenaga, Y. Tamai, K. Okumura, K. Ishida, K. Sawada, Label-free acetylcholine image sensor based on charge transfer technology for biological phenomenon tracking, Japanese Journal of Applied Physics 51, 027001 (2012); doi: 10.1143/JJAP.51.027001

[5] F. Mizutani, S. Yabuki, Y. Hirata, Amperometric Ilactate-sensing electrode based on a polyion complex layer containing lactate oxidase. Application to serum and milk samples, Analytica Chimica Acta 314, 233-239 (1995); doi: 10.1016/0003-2670(95)00278-8

[6] D. E. Yates, S. Levine, T. W. Healy, Site-binding model of the electrical double layer at the oxide/water interface, Journal of the Chemical Society, Faraday Transactions 170, 1807-1818 (1974); doi: 10.1039/F19747001807

[7] A. Poghossian, M. J. Schöning, Silicon-based chemical and biological field-effect sensors, Encyclopedia of Sensors Vol. X, American Scientific Publishers, 1-71 (2006)

[8] T. Yoshinobu, H. Iwasaki, Y. Ui, K. Furuichi, Y. Ermolenko, Y. Mourzina, T. Wagner, N. Näther, M. J. Schöning, The light-addressable potentiometric sensor for multi-ion sensing and imaging, Methods 37, 94-102 (2005); doi: 10.1016/j.ymeth.2005.05.020

[9] J. R. Siqueira Jr., C. F. Werner, M. Bäcker, A. Poghossian, V. Zucolotto, O. N. Oliveira Jr. and M. J. Schöning, Layer-by-layer assembly of carbon nanotubes incorporated in light-addressable potentiometric sensors, Journal of Physical Chemistry C 113, 14765-14770 (2009); doi: 10.1021/jp904777t

[10] C. F. Werner, S. Schusser, H. Spelthahn, T. Wagner, T. Yoshinobu, M. J. Schöning, Fieldprogrammable gate array based controller for multi spot light-addressable potentiometric sensors with integrated signal correction mode, Electrochimica Acta 56, 9656-9660 (2011); doi: 10.1016/j.electacta.2011.03.012

[11] T. Wagner, R. Molina, T. Yoshinobu, J. P. Kloock, M. Biselli, M. Canzoneri, T. Schnitzler, M. J.
Schöning, Handheld multi-channel LAPS device as a transducer platform for possible biological and chemical multi-sensor applications, Electrochimica Acta 53, 305-311 (2007);

doi: 10.1016/j.electacta.2007.04.006 